\title{
Histone tales: echoes from the past, prospects for the future
}

\author{
Chris Murgatroyd and Dietmar Spengler*
}

\section{Abstract \\ Recent studies of the nematode dauer state provide new insights into epigenetic processes that underlie cellular memory.}

It has been 150 years since Charles Darwin described in his seminal work On the Origin of Species how descent with modification and natural selection could explain the diversity of life. When Mendel's theory of inheritance was rediscovered in the early 1900 s and verified to be consistent with natural selection, evolutionary biologists adopted genetics as the central pillar of the Modern or Neo-Darwinian Syntheses and committed to genetics as the center of their explanatory paradigm. With Darwinism becoming widespread, the discovery of the DNA double helix structure by Watson and Crick was hailed as finally delivering the long-sought hereditary mechanisms for evolutionary theory.

The concentration on genetics has now lasted almost a century and, despite some claims to the contrary, evolutionary genetics established the consistency, though not the sufficiency, of genetics and natural selection to explain evolution. We are now coming to realize that gene-centric theories of evolution are limited in their scope [1,2]. This shortcoming has been addressed by the life history theory, which analyzes the evolution of whole-organism traits (in particular phenotypic variations such as size at birth, growth rates, age and size at maturity, clutch size and reproductive investment, mortality rates and lifespan) on the basis of the criteria that life histories are shaped by the interaction of extrinsic and intrinsic factors. It states that extrinsic factors are ecological impacts on survival and reproduction and that intrinsic factors are tradeoffs among life history traits and lineage-specific constraints on the expression of genetic variation.

*Correspondence: spengler@mpipsykl.mpg.de

Department of Molecular Neuroendocrinology, Max Planck Institute of Psychiatry, Kraepelinstrasse 2-10, D-80804 Munich, Germany
Although life history theory can offer detailed answers to the question of why phenotypes are different, it does not attempt to understand the mechanisms that mediate and integrate these differences. Three recent studies [3-5] take us, however, an important step forward in this direction. They build on the nematode Caenorhabditis elegans as a unique model system for studying animal survival strategies and the environmental regulation of life histories. C. elegans is a rapidly growing worm and its entire life cycle, from an egg to an adult, takes only 3.5 days in the presence of abundant food at $20^{\circ} \mathrm{C}$. After embryogenesis, C. elegans progresses through four larval stages (L1 to L4) before becoming an adult (Figure 1). When developing C. elegans larvae sense the pheromone daumone (a mixture of ascarosides), they enter into a physiologically specialized state - the dauer state - that is capable of long-term survival.

Genetic studies have identified various signaling systems as critical regulators of dauer state, including insulin and transforming growth factor- $\beta$ signaling. Moreover, two G-protein-coupled chemoreceptors (srbc-64 and srbc-66) have been recently identified to mediate the effect of the dauer hormone [3]. Although the dauer stage is a well-known option for escaping unfavorable conditions for young larvae, animals at later stages use different means to cope with the environment. In fact, the dauer stage of $C$. elegans is not only a survival strategy, but also induces adult reproductive diapause, a delay of reproduction that results in increased longevity and protection of germ stem cells. Adult diapause entry and exit are regulated by the gene nhr-49 [4], which encodes a nuclear hormone receptor, and daf-12, another member of this gene family, controls dauer formation.

Together with previous work, these two studies $[3,4]$ deepen our insight into critical regulatory nodes of larval dauer and adult diapause, although they do not reveal the integrated responses that presumably underpin these states. In a recent study, however, Hall and coworkers [5] report a refreshing approach to the topic by performing a series of well-designed experiments to unravel the complexity of the gene-environment dialog for different life histories at an organismal scale. Genome-wide expression profiling for control and postdauer whole 


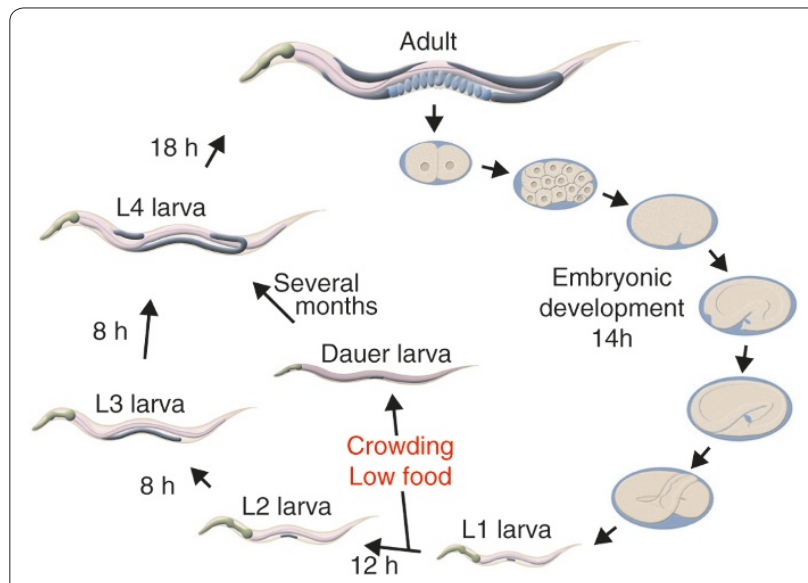

Figure 1. The life cycle of $C$. elegans. Under favorable conditions animals pass through direct development to adulthood in as little as 3 to 4 days. In response to harsh environmental conditions, such as food shortage, crowding or high temperatures, animals can enter into an arrested dauer stage.

adult animals led to the identification of over 2,000 genes that were significantly upregulated in either group (one of the largest groups being associated with reproduction). By extending their analysis to dauer, postdauer and control larvae they could further show that the altered gene expression observed in postdauer animals arises from multiple regulatory mechanisms acting during the dauer stage (164 genes), on subsequent resumption of reproductive growth (143 genes) or at both stages (400 genes). Functionally, these changes correlate with a longer mean life span and a larger brood size in the postdauer stage animals. Although the authors [5] do not directly demonstrate which of these genes causes the phenotype, they conclude that differences in life history have been hard-wired at the level of persistent changes in gene expression patterns. So what are then the molecular mechanisms that couple differences in life histories to gene expression patterns that drive the development of different phenotypes?

Epigenetic regulation of gene expression allows the integration of intrinsic and environmental signals in the genome and can facilitate the adaptation of organisms to changing environments through alterations in gene expression [6]. DNA methylation is one of the most intensively studied epigenetic mechanisms, with an established role in development and cellular differentiation. However, the global DNA methylation pattern seen in vertebrates is by no means ubiquitous among eukaryotes. Several wellstudied model systems, including C. elegans, have no recognizable Dnmt-like genes and are devoid of DNA methylation. This prompted the authors [5] to investigate whether, alternatively, histone modifications could bridge the dauer state to a lasting transcriptional record.
Genome-wide analysis by chromatin immunoprecipitation followed by sequencing (ChIP-seq) showed that chromatin marks associated with euchromatin (panacetylation of histone $\mathrm{H} 4, \mathrm{H} 4 \mathrm{ac}$, and trimethylation of histone $\mathrm{H} 3$ at lysine 4, H3K4me3) were decreased in postdauer animals, despite similar overall gene expression levels, and localized primarily to highly expressed genes [5]. In contrast, repressive chromatin marks (H3K9me3 and H3K27me3) showed similar levels in control and postdauer animals. In support of a functional role for these changes, active but not repressive marks correlated positively with gene expression. Importantly, this global histone signature also extended to the genes that were altered in postdauer animals. Euchromatic marks were mainly reduced at upregulated genes but less at downregulated ones and correlated positively with gene expression. Intriguingly, however, no correlation was observed between the fold change in gene expression and the chromatin modification profiles between control and postdauer populations.

Together, these data show that exposure to dauer state leaves a deep trace on the epigenome, which manifests as a genome-wide loss of active chromatin marks. Given that this kind of cellular memory primed only some but not all genes for subsequent changes in their expression, additional locally acting mechanisms seem to be at work. In a nutshell, the dauer-induced chromatin modeling did not lead per se to changes in gene expression but paved the way for these changes on a site-specific scale.

To test the hypothesis that dauer-induced chromatin changes can act as a 'pacemaker' for changes in local transcription, the authors [5] studied a series of $C$. elegans mutants with defects in different components controlling chromatin assembly (histone deacetylases, chromatin remodeling ATPase, chromatin associated proteins, and others). Although these mutations did not interfere with dauer formation, they affected to varying degrees and specificity the dauer-induced regulation of two genes that were studied as examples (the Major Sperm Protein family member gene $m s p-64$, and the choline/carnitine O-acyltransferase gene W03F9.4). These data strongly suggest that dauer-induced chromatin remodeling is necessary, but not sufficient, for cellular memory formation in C. elegans.

Collectively, the results from Hall and coworkers [5] suggest a two step model in the formation of a cellular memory underpinning life history traits in C. elegans (Figure 2). In this model, global changes in histone modifications exert a universal gatekeeper function by preparing the ground for rewiring developmental trajectories in response to environmental stimuli. Although these chromatin marks seem to be central to the formation of a kind of rudimentary memory, they do not suffice to control the overall transcriptome. However, 


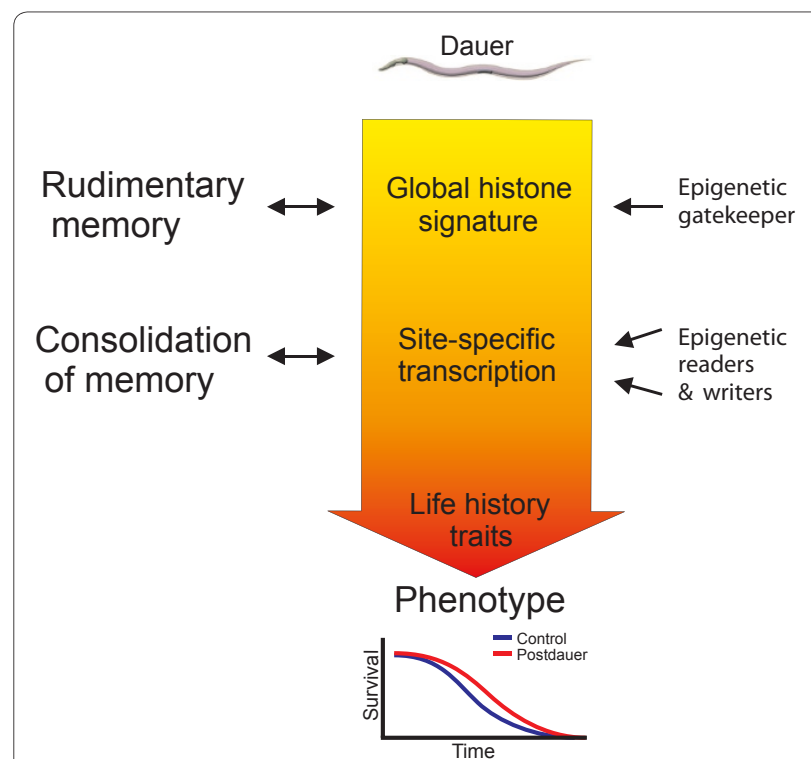

Figure 2. The gatekeeper function of histones in cellular memory. The dauer state of C. elegans induces global changes in histone marks that are necessary, but not sufficient, for the formation of a cellular memory that governs life history traits. The dauer-dependent histone signature serves as a kind of rudimentary memory that prepares the ground for further downstream sitespecific changes in gene transcription, which lead to a consolidation of the memory process. The epigenetic mechanisms that read the dauer signature, rewrite local histone marks and control transcriptional factors that together induce local changes in gene expression are currently unknown. Ultimately, these transcriptional events trigger altered developmental trajectories that underpin life history traits and manifest with distinct phenotypes.

by resetting the epigenomic activation pattern, they facilitate further downstream, currently unknown, mechanism(s) to index genes in a site-specific manner. Therefore, dauer-induced genome-wide changes in histone modifications seem to be permissive for the rewiring of gene expression patterns that serve to consolidate memory formation.
Future studies are necessary to elucidate in greater detail how permissive (global) and directive (site-specific) mechanisms are interconnected in cellular memory formation and whether distinct environmental signals operate at these different scales. The findings [5] also provoke the question of whether such graded memory formation in response to dauer state reflects a general principle of life history trait formation in C. elegans. If this is the case, it may represent an ancient and possibly conserved mechanism that applies in concert with DNA methylation to other eukaryotes and could underpin processes in cellular memory of profound socioeconomic and medical implications $[7,8]$. In any case, the availability of powerful tools such as ChIP-seq and RNA interference make C. elegans an excellent model organism for shedding new light on the role of chromatin in memory formation and life history traits.

Published: 26 February 2010

\section{References}

1. Jablonka E, Lamb MJ: Evolution in Four Dimensions: Genetic, Epigenetic, Behavioral and Symbolic Variation in the History of Life. 1st edition. Cambridge, MA: MIT Press; 2005

2. Cloud J: Why your DNA isn't your destiny. TIME 2010, 18:49-53. [http://www time.com/time/health/article/0,8599,1951968,00.html]

3. Kim K, Sato K, Shibuya M, Zeiger DM, Butcher RA, Ragains JR, Clardy J, Touhara K, Sengupta P: Two chemoreceptors mediate developmental effects of dauer pheromone in C. elegans. Science 2009, 326:994-998.

4. Angleo G, Van Gilst MR: Starvation protects germline stem cells and extends reproductive longevity in C. elegans. Science 2009, 326:954-958.

5. Hall SE, Beverly M, Russ C, Nusbaum C, Sengupta P: A cellular memory of developmental history generates phenotypic diversity in C. elegans. Curr Biol 2010, 20:149-155.

6. Jaenisch R, Bird A: Epigenetic regulation of gene expression: how the genome integrates intrinsic and environmental signals. Nat Genet 2003, 33 Suppl:245-254.

7. Gluckman PD, Hanson MA: Living with the past: evolution, development, and patterns of disease. Science 2004, 305:1733-1739.

8. Zhang TY, Meaney MJ: Epigenetics and the environmental regulation of the genome and its function. Annu Rev Psychol 2010, 61:439-466.

doi:10.1186/gb-2010-11-2-105

Cite this article as: Murgatroyd C, Spengler D: Histone tales: echoes from the past, prospects for the future. Genome Biology 2010, 11:105. 